\title{
EXPERIENCIA E INICIACIÓN COMPETITIVA DE LOS ESCOLARES PARTICIPANTES EN LOS IV JUEGOS DEPORTIVOS CENTROAMERICANOS Y DEL CARIBE, COLOMBIA
}

\author{
EXPERIENCE AND COMPETITIVE INITIATION OF SCHOOL PARTICIPANTS \\ IN THE IV JUEGOS DEPORTIVOS CENTROAMERICANOS Y DEL CARIBE, COLOMBIA
}

\author{
Luis Gerardo Melo Betancourt ${ }^{1}$ \\ Hilario Moreno Bolívar ${ }^{2}$ \\ Luisa Fernanda Gutiérrez ${ }^{3}$
}

\section{RESUMEN}

Con el objetivo de caracterizar el historial competitivo y la iniciación en los procesos de formación deportiva de los escolares participantes en los IV Juegos Deportivos Escolares Centroamericanos y del Caribe 2013, en la ciudad de Armenia, Colombia, se realizó un estudio cuantitativo con alcance descriptivo y corte transversal. De 683 individuos asistentes se tomó una muestra intencional de 233 escolares, provenientes de 9 países en 12 modalidades deportivas, con edad promedio de 16,9 y 16,8 años en hombres y mujeres, respectivamente. La técnica utilizada fue la encuesta, y se implementó tanto en los sitios de competencia como de concentración. Se encontró que el inicio de la formación deportiva comenzó a los 9,5 años de edad $+3,2$ y el de competencia a los 10,5 años. El $95 \%$ de los encuestados tiene experiencia en competencias internacionales y, en el último año de preparación, compitieron en 52 torneos antes de asistir al evento. Los escolares participantes en los Juegos Intercolegiados Centroamericanos y del Caribe, tienen procesos formativos y competitivos de deportistas de alto rendimiento.

Palabras claves: escolares, iniciación y experiencia deportiva, juegos, Centroamérica.

\section{Abstract}

In order to characterize the competitive history and initiation processes sports training school participate in the IV School Sports Games Central American and Caribbean 2013 in the city of Armenia Colombia, a quantitative cross-sectional study was performed with descriptive scope and of 683 individuals attending a purposive sample of 233 students, from 9 countries in 12 sports categories, with an average age of 16.9 and 16.8 years in men and women respectively was taken. The technique used was the survey being applied both sites of competition and concentration. It was found that the onset of sports training began at age 9.5 years and competition +3.2 to 10.5 years. $95 \%$ of respondents have experience in international competitions and last year had 52 competitions preparation before attending the event. Schools participate in the Intercollegiate Central American and Caribbean Games have training and competitive processes for high performance athletes.

Keywords: school, initiation and sports experience, intercollegiate games.

1 Ph.D en Entrenamiento Deportivo; licenciado en Educación Física. Docente Universidad de Caldas. Correo electrónico: luis.melo@ucaldas.edu.co

2 Magíster en Intervención Integral en el Deportista, licenciado en Educación física. Docente Universidad del Quindío. Correo electrónico: hmoreno@ uniquindio.edu.co

3 icenciada en Educación Básica con Énfasis en Educación Física. Docente Politécnico Nacional Manizales. Correo electrónico:lufegu23@hotmail.com 
Fecha de recepción: 15 de mayo de 2015

Fecha de aprobación: 1 de octubre de 2015

Para citar este artículo:

Melo, L.G.; Moreno, H. y Gutiérrez, L.F. (2015). Experiencia e iniciación competitiva de los escolares participantes en los IV Juegos Deportivos Centroamericanos y del Caribe, Colombia. Lúdica Pedagógica, (22), 69-74.

\section{INTRODUCCIÓN}

Según la National Association for Sport and Physical Education (NASPE) (2010), de Estados Unidos, el deporte infantil y juvenil en los últimos años se ha convertido en centro de intereses de muchas instituciones gubernamentales estatales y privadas, en diversos países del mundo, encargadas tanto de educar como de preparar deportistas desde niveles de iniciación, hasta el alto rendimiento. La preocupación de la mayoría de naciones es depurar y desarrollar los procesos conducentes hacia la formación de deportistas, con la estructuración de programas a corto y mediano plazo que les permita figurar de alguna forma en el contexto nacional e internacional.

En este sentido, la NASPE (2010) alude que el desarrollo de los atletas de élite y la motivación para hacer deporte toda la vida se promueven con mayor eficacia cuando los jóvenes participan en juegos informales durante la infancia y también cuando se desempeñan en diversos deportes hasta los 15 años de edad. Esto facilita el desarrollo social entre los jóvenes y expande sus intereses, identidades y experiencias, proporcionando una base para la elección de un determinado deporte en etapas posteriores.

En correspondencia con lo anterior, Platonov (1994) propone que desde edades tempranas se debe vincular a los escolares en procesos permanentes de entrenamiento, como es el caso de la natación. Según este autor, la adaptación a largo plazo del organismo a las cargas de entrenamiento y de competición está supeditada a la edad de inicio en la práctica deportiva y a los años de entrenamiento necesarios para lograr los mejores resultados deportivos. La mayoría de nadadores del mundo inician sus entrenamientos entre los 8 y 12 años, en los hombres, y entre los 6 y 10 años, en las mujeres (Platonov, 2001).

Un estudio con atletas cubanos (Romero, 2003) demostró que los altos rendimientos deportivos en la edad infantil y juvenil son una garantía fehaciente de eleva- dos éxitos deportivos en el futuro; aunque también se ha evidenciado, que uno o dos años después de haber rebasado la edad juvenil, se encuentran deportistas exitosos que a la edad juvenil no habían figurado entre los mejores.

En Colombia, de acuerdo con Coldeportes (2009), en el Plan Decenal del Deporte 2009-2019, que contiene la política pública del sector de la Educación Física, la Recreación, el Deporte y la Actividad Física para la Salud, estableció lineamientos hacia la orientación y masificación de las actividades físicas y el deporte escolar. En ese sentido, los Juegos Intercolegiados se definen como una estrategia principal del Sistema Nacional del Deporte para promover y articular la detección temprana de talentos, especializar la práctica, focalizar y priorizar la intervención estatal y privada en esa materia; para ello, se debe estimular y apoyar la realización sistemática de los Juegos Intercolegiados y de los Juegos Universitarios, donde las decisiones de política deben privilegiar los procesos de perfeccionamiento, cualificación y competición de los mejores deportistas, hasta hacerlos parte del deporte asociado y de los calendarios deportivos, regionales, nacionales e internacionales.

Los Juegos Intercolegiados Centroamericanos y del Caribe son un programa de política Internacional descentralizada, que permite que cada nación, de manera autónoma, ajuste, regule y promueva los procesos que impulsen la práctica del deporte escolar en los centros educativos, centros de formación, ligas deportivas y clubes deportivos, de acuerdo con sus intereses y prioridades de las prácticas deportivas que están enmarcadas en los diferentes programas de los planes y programas del deporte en cada país.

Por consiguiente, el objetivo que orientó el estudio fue caracterizar el historial competitivo y la iniciación en los procesos de formación deportiva de los escolares 
participantes en los IV Juegos Deportivos Escolares Centroamericanos y del Caribe 2013, en la ciudad de Armenia, Colombia.

\section{MATERIAL Y MÉTODOS}

Tipo de estudio

Dentro del enfoque cuantitativo, se desarrolló un estudio descriptivo de corte transversal.

\section{Población y muestra}

De 683 deportistas (339 hombres y 345 mujeres) participantes en los IV Juegos Deportivos Escolares Centroamericanos y del Caribe Armenia 2013, se encuestaron 233 seleccionados por conveniencia, de los cuales 102 fueron hombres y 131 mujeres, en representación de 9 países participantes y de 12 modalidades deportivas.

\section{PROCEDIMIENTO}

Inicialmente se elaboró una encuesta por el equipo investigador, la cual fue sometida a juicio de expertos de diversas ligas deportivas, profesionales de la educación física y los deportes en diferentes universidades en Colombia; posteriormente se ajustó a partir de las sugerencias y se le realizó a un grupo piloto de deportistas del departamento de Caldas y, así, se construyó la propuesta definitiva, la cual se implementó en la recolección de la información. Las encuestas fueron ejecutadas por colaboradores capacitados con anterioridad. Estas se llevaron a cabo tanto en los sitios de competencia como en los alojamientos, de forma personal entre los días 10 y 15 de noviembre de 2013, en la ciudad de Armenia,Colombia.

La encuesta contenía las siguientes preguntas:

¿A qué edad inició la práctica de su deporte? ¿A qué edad tuvo su primera competencia deportiva? ¿Cuál es el nivel máximo en el que ha competido: mundial; panamericano, centroamericano, suramericano o nacional? ¿En cuántas competencias participó en el último año? ¿De estas, cuántas eran preparatorias? ¿Cuántas eran principales?
Para el análisis estadístico se elaboró una base de datos en Excel 2010, para ser tabulados y luego procesados en el programa estadístico SSPS (v. 15.0), para posteriormente ser presentados en tablas de frecuencia, promedio y desviación estándar.

\section{CONSIDERACIONES BIOÉTICAS}

La participación de los deportistas fue voluntaria, y se les garantizó el anonimato y la confidencialidad de sus respuestas. Una vez autorizaban responder la encuesta, firmaban el consentimiento informado, según lo reglamentado en la Resolución 8430 de 1993, artículo 11, emanado del Ministerio de Salud, dado que la solución del instrumento no implicaba ningún riesgo para los participantes del estudio.

\section{RESULTADOS Y DISCUSIÓN}

\section{Características sociodemográficas \\ de la población evaluada}

Fueron encuestados 233 deportistas provenientes de 9 países (Colombia, México, Guatemala, Panamá, Venezuela, República Dominicana, Nicaragua, Puerto Rico y Costa Rica) y de 12 modalidades deportivas (voleibol, natación, lucha, atletismo, triatlón, halterofilia, taekwondo, tenis de mesa y de campo, judo, patinaje y baloncesto). La edad media fue de $16,94( \pm 0,78)$ años, para los hombres, y de $16,81( \pm 0,75)$, para mujeres, con una mínima de 15,10 y una máxima de 18,18.

Tabla 1. Promedio y desviación estándar de la edad de iniciación y competición deportiva de los deportistas escolares evaluados (años).

\begin{tabular}{|l|c|c|}
\hline \multicolumn{1}{|c|}{ Variable } & Promedio & Desviación \\
\hline $\begin{array}{l}\text { Edad en la que inició la } \\
\text { práctica de su deporte }\end{array}$ & 9,5 & 3,2 \\
\hline $\begin{array}{l}\text { Edad en la cual inició las } \\
\text { competencias deportivas }\end{array}$ & 10,5 & 2,1 \\
\hline
\end{tabular}

Fuente: elaboración de los autores.

Como se puede apreciar en la tabla 1, los escolares iniciaron en promedio su práctica deportiva un poco antes de los diez años, ante lo cual, según Blázquez (1999), existen diferentes opiniones sobre la edad óptima para 
comenzar el aprendizaje deportivo y competitivo. El autor plantea que entre los 9 y 11 años son las edades apropiadas para la iniciación deportiva, aunque es posible que se pueda adelantar en 5 años en algunos deportes individuales. También propone que si alguien comienza antes o después de estas edades, los rendimientos futuros no alcanzarán un nivel excepcional.

Contrariamente, Castro (2000) propone que la edad en la cual se debe iniciar la formación deportiva es entre los 7 y 8 años, ya que dicho proceso no comienza cuando el niño tiene contacto directo con el deporte específico, sino durante su formación básica, en la cual se adquieren habilidades de base para posteriores aprendizajes motrices específicos y más complejos. También existen otros lineamientos de iniciación deportiva según las características del deporte. Por ejemplo, Ruiz, García y Casimiro (2001) plantean que para los deportes de pelota, como el balonmano, fútbol, voleibol, baloncesto, entre otros, se debe iniciar entre los 10 y los 13 años; para Durán (1988), en béisbol y el softbol se debe comenzar durante 5 y 8 años de edad; tenis a los 8 ; lucha a los 6; natación y gimnasia a los 4 y 5 .

Algo indiscutible en los procesos de formación deportiva, independientemente de los planteamientos teóricos antes citados, es que según la proyección y preferencia de cada infante, algunos procesos de iniciación se deben dar más tempranamente que otros, ya que hay disciplinas atléticas que demandan unas características antropométricas y motrices condicionales muy específicas para lograr buenos resultados, como el caso de la gimnasia artística y la natación entre otros, en los cuales la especialización deportiva debe ser entre los 6 y 7 años (Ramos, Melo y Alzate, 2007).

Respecto a los resultados concernientes al inicio de la parte competitiva, este fue un fenómeno que se dio tempranamente (10,5 años), lo cual es favorable para los procesos de formación de alto rendimiento, según Hahn (1988), quien argumenta que comenzar prematuramente la competencia motiva a los entrenadores a formar hacia el alto rendimiento, en búsqueda del éxito precoz. En contraposición a estos sustentos, tanto Blázquez (1999) como Fröhner (2003) afirman que comenzar la competencia deportiva temprana acarrea riesgos físicos como: los óseos y articulares, cardiacos y musculares; riesgos psicológicos en cuanto a la conducta del sujeto y al estado mental, que arrojan como resultado niveles altos de ansiedad, estrés, frustración, riesgos y abandonos deportivos, como también, la introducción en prácticas deportivas para los cuales no tienen condiciones especiales.

Tabla 2. Nivel máximo de competencia que han tenido en su historial deportivo los escolares evaluados.

\begin{tabular}{|l|c|c|}
\hline Tipo de campeonato & $\begin{array}{c}\text { Número de } \\
\text { deportistas }\end{array}$ & Porcentaje (\%) \\
\hline Mundial & 84 & 36 \\
\hline Panamericano & 61 & 26 \\
\hline Centroamericano & 53 & 23 \\
\hline Suramericano & 23 & 10 \\
\hline Nacional & 12 & 5 \\
\hline
\end{tabular}

Fuente: elaboración de los autores.

Como se aprecia en la tabla, el $95 \%$ de los escolares encuestados tiene experiencia en competencias internacionales, destacándose que un $36 \%$ ha competido en el ámbito mundial. Esto indica que los escolares participantes en dichos eventos tienen una trayectoria competitiva considerable, entendiendo por competición, la totalidad de las medidas adecuadas que capacitan al deportista para obtener rendimientos deportivos óptimos (Weineck, 2005). Al respecto, Ruiz, García y Casimiro (2001) argumentan que la supuesta excelencia que genera la actividad competitiva, en la práctica produce un énfasis en el elitismo y en la superioridad respecto al prójimo, convirtiendo desde tempranas edades la búsqueda de la victoria a toda costa.

De acuerdo con Manno (1994), para llegar a competir en torneos importantes del carácter nacional e internacional, se requieren de 12 a 15 años de formación, tiempo necesario para el logro de los niveles de preparación, por lo tanto, los deportistas deberían iniciar a competir en los torneos de importancia nacional e internacional entre los 16 y 18 años de edad. De acuerdo con lo planteado, los deportistas del presente estudio iniciaron prematuramente los procesos de competencia nacional e internacional, ya que pese a tener en promedio 16 años de edad, preliminarmente a la clasificación para los Juegos Centroamericanos y del Caribe 2013, el 95 \% de ellos ya había participado en diversas justas deportivas de índole mundial. 
Sin embargo, no se debe desconocer que los procesos de entrenamiento del deporte de alto rendimiento son prolongados y se reflejan a largo plazo; es decir que todos los factores implícitos en la preparación para el alcance de los objetivos trazados, inicien a edades tempranas, dentro de ellos la competencia deportiva, que si bien no tiene que ser necesariamente a nivel internacional, si se requiere para el afianzamiento técnico, táctico y psicológico entre otros aspectos.

Tabla 3. Número de competencias en las que han participado en el último año los deportistas escolares evaluados.

\begin{tabular}{|l|c|c|}
\hline $\begin{array}{c}\text { Tipo de } \\
\text { competencia }\end{array}$ & Cantidad & Porcentaje (\%) \\
\hline Preparatorias & 40 & 76,9 \\
\hline Principales & 12 & 23,1 \\
\hline
\end{tabular}

Fuente: elaboración de los autores.

De acuerdo con los resultados registrados en la tabla 3, el mayor porcentaje de competencias que tuvieron los evaluados durante el último año antes de asistir a los Juegos Intercolegiados Centroamericanos, se orientó al componente de preparación; teniendo en cuenta que dentro del calendario de competencias se encuentran desde las regionales, nacionales e internacionales. Tanto para Martin, Nicolaus, Ostroski y Rost (2004) como para Navarro, Oca y Rivas (2010), el número de competencias preparatorias de un deportista varía según la edad, objetivos, tipo de deporte, presupuesto y rendimiento, entre otros aspectos. Queda claro, entonces, que en los últimos años especialmente las competencias de los jóvenes, han recibido un incremento sustancial por la ampliación de las ofertas en las disciplinas deportivas, la diversificación de los programas de competición de las asociaciones, aumento del grado de organización de los sistemas de competencia específicos de la disciplina deportiva y el claro descenso de la edad de incorporación al deporte competitivo.
Por lo anterior, Issurin (2012) sustenta los cambios que han surgido en el mundo del deporte durante las últimas décadas, especialmente en el aumento drástico del número de competiciones durante toda la temporada, ya que deportistas internacionales de deportes como ciclismo de carrera pasó de 38 a 48 días; lucha libre de 25 a 30 ; judo de 12 a 20; esgrima de 15 a 20, y natación de 25 a 35. Estos guarismos concuerdan con la cantidad de competencias que afrontaron los escolares que participaron en los Juegos Centroamericanos y del Caribe en el último año, pues un escolar que no es un deportista profesional tenga que afrontar 52 competencias en el año, es una cantidad monumental.

\section{CONCLUSIONES}

Si bien es cierto que el deporte escolar tiene una connotación formativa a nivel integral o biopsicosocial de acuerdo a las políticas gubernamentales y educativas de las diversas naciones, a través del cual se fomente la práctica deportiva, la creación de hábitos de ejercicio, la integración y convivencia ciudadana, detección de talentos deportivos, fortalecimiento de valores como el respecto, tolerancia, trabajo en equipo, el asumir el triunfo y la derrota como procesos inherentes al desarrollo humano como para citar sólo algunos aspectos; el perfil formativo y competitivo de la población de estudio, es de deportistas de alto rendimiento.

Las instituciones educativas que pretendan llegar a competir en instancias de juegos intercolegiados de índole internacional, como la del presente estudio, deben orientar y desarrollar los procesos deportivos de los educandos hacia el alto rendimiento deportivo, o articularlos con aquellos orientados por ligas y clubes deportivos de forma mancomunada; pues es muy complejo - casi utópico-que un escolar solamente con los procesos normales de educación física que suelen ser de dos horas semanales y no de forma específica hacia el deporte, llegue a instancias de competencias internacionales, para lo cual se requiere de procesos rigurosos, específicos y planificados a largo plazo. 


\section{REFERENCIAS BIBLIOGRÁFICAS}

Blázquez, D. (1999). La iniciación deportiva y el deporte escolar. Barcelona: INDE.

Castro, U. (2000). La iniciación a los deportes desde su estructura y dinámica: aplicación a la educación física escolar y el entrenamiento deportivo. Barcelona: INDE.

Coldeportes (2009). Plan Decenal del Deporte, la Recreación, la Educación Física y la Actividad Física para el Desarrollo Humano, la Convivencia y la Paz 2009-2019. Bogotá.

Durán, M. (1988). El niño y el deporte. Barcelona: Paidós.

Fröhner, G. (2003). Esfuerzo físico y entrenamiento en niños y jóvenes. Barcelona: Paidotribo.

Hahn, E. (1988). Entrenamiento con niños. Barcelona: Martínez Roca.

Issurin, V.B. (2012). Entrenamiento deportivo. Periodización en bloques. Barcelona: Paidotribo.

Mano, R. (1994). Fundamentos del entrenamiento deportivo. Barcelona: Paidotribo.

Martin, D.; Nicolaus, J.; Ostroski, C.H. y Rost, K. (2004). Metodología general del entrenamiento infantil $y$ juvenil. Barcelona: Paidotribo.

National Association for Sport and Physical Education (NASPE) (2010). Guidelines for participation in youth sport programs: Specialization versus multi- sport participation. Recuperado de http://www. shapeamerica.org/advocacy/positionstatements/pe/ loader.cfm?csModule=security $/$ getfileypageid $=4651$

Navarro, F.; Oca A. y Rivas, A. (2010) Planificación del entrenamiento y su control. Madrid: Cultiva Libros.

Platonov, V. (1994). Los sistemas de entrenamiento de los mejores nadadores del mundo. Barcelona: Paidotribo.

Platonov, V. (2001). El entrenamiento deportivo. Teoría y metodología. Barcelona: Paidotribo.

Ramos, S.; Melo L. y Alzate, D. (2007). Evaluación antropométrica y motriz condicional de niños $y$ adolescentes. Manizales: Universidad de Caldas.

Romero, E. (2003). La clasificación deportiva en el atletismo juvenil cubano. Efdeportes.com 8(57). Recuperado de: http://www.efdeportes.com/efd57/ atl1.htm

Ruiz, F.; García, A. y Casimiro, A. (2001). La iniciación deportiva basada en los deportes colectivos. Madrid: Gymnos.

Weineck, J. (2005). Entrenamiento total. Barcelona: Paidotribo. 